\title{
State estimation of the Tanzanian power system network using non-quadratic criterion and MATLAB environment
}

\author{
Mashauri Adam Kusekwa \\ Electrical Engineering Department, Dar es Salaam Institute of Technology,Dar es Salaam, Tanzania
}

Email address:

kusekwa_adam@yahoo.com, kusekwa_adam@dit.ac.tz

\section{To cite this article:}

Mashauri Adam Kusekwa. State Estimation of the Tanzanian Power System Network Using Non-Quadratic Criterion and MATLAB Environment. International Journal of Energy and Power Engineering. Vol. 3, No. 5, 2014, pp. 266-276. doi: $10.11648 /$ j.ijepe.20140305.18

\begin{abstract}
Power system state estimation is an effective online tool for monitoring, control and for providing consistent database in energy management systems. This paper presents an algorithm for state estimation of the Tanzanian power system network using a non-quadratic state criterion. Equality and inequality constraints existing in a power system are included in formulating the estimation problem. Equality constraints are target values used in load flow analysis and are included in power system state estimation in order to restore observability to those parts of the power system network which are permanently or temporarily unobservable. Inequality constraints are limits such as minimum and maximum reactive power generation, transformer tap and phase-shift. The solution techniques used is primal-dual interior point logarithmic barrier functions to treat the inequality constraints. An algorithm is developed using the method and a program coded in MATLAB is applied in implementing the simulation. Computational issues arising in the implementation of the algorithm are presented. The simulation results demonstrate that the primal-dual logarithmic barrier interior point algorithm is a useful numerical tool to compute the state of an electrical power system network. The inequality constraints play essential role in enhancing the reliability of the estimation results. Also, it is expected that significant benefit could be gained from application of the constrained state estimation algorithm to the Tanzanian power system network.
\end{abstract}

Keywords: Power Systems, Non-Quadratic State Estimation, Simulation, Interior Point Method, MATLAB Program

\section{Introduction}

Power system state estimation is a mathematical procedure [1] which processes a set of real-time measurements such as voltages, real and reactive power injections, real and reactive power flows using the topology determined by the topology processor to come out with the best estimate of the current state of the power system. The state of a system is defined as a vector of the voltage magnitude and voltage angle of each bus of that system.

Power system state estimation is important for control and security monitoring of a system. Using real-time system measurements, it is easy to identify whether the system is normal or not. In addition, the state estimator is used to build the model for the observable part of the network [2]. It is used to filter redundant data [2-3] to eliminate incorrect measurement, and to produce reliable state estimates.

Before any control action can be taken or security assessment can be made, a reliable estimate of the current state of the system must be determined. For this purpose the number of physical measurements cannot be restricted to only those quantities required to support the load flow computations. The input to the load flow studies is confined to real and reactive power injections at load buses, and real power and voltage magnitude at voltage-controlled buses. If one of these inputs is unavailable, the load flow solution cannot be determined. In addition, gross-error in one of the input quantities can cause the load flow solution to be useless. In practice, other conveniently measured quantities such as real and reactive power flows are available, but they cannot be used in the load flow program. These limitations can be removed by state estimation.

The main objective of the state estimator is to find best estimate of unknown voltage angle at every bus in the modelled system network [4]. Since inexact measurementssuch as those from SCADA system are used to calculate the state vector, the estimate will also be inexact. This introduces the problem of how to device the best estimate for the state 
vector given the available measurements.

The results from state estimation provides the real-time database [1],[3] for other network applications such as security assessment, determination of power flows in parts of network that are not directly metered, optimal power flow, contingency analysis, etc. State estimation can also be used for data validation. One of the major benefits of state estimation is its ability for detection and identification of bad data.

The technical literature is rich pertaining to power system state estimation. The pioneering work is due to Schweppe et al [5-7]. The first practical implementation of state estimator is reported in [8]. The model for state estimation problem is well established and diverse solutions are also well known. Appropriate background information on power system state estimation can be found in [9], [2], and [10].

Particularly interesting is the work by Abur et al [11-12]. The work reported in [11] implements a fast algorithm for the weighted least absolute value (WLAV) state estimation using simplex method. WLAV fall under non-quadratic criterion. This work is extended in [12] to include equality and inequality constraints on measurements. Incorporating of constraints in formulating state estimation problem enhances the reliability of the estimator. In all the described work the state estimation problem is formulated as a linear programming (LP) problem and solved by using simplex method. Interest of using interior point method is described in the work of Clements et al [13]. Application of interior point method is extended in [14-15]. The work reported in [13], [14] is different from the work described in this paper. In [13] logarithmic barrier function is directly used in solving the problem. In [14] the authors formulated the state estimation problem as an optimization problem and used the barrier function method in solving the primal formulation and affine scaling method in solving the dual formulation of the problem.

In this paper, the problem is set up as an optimization problem with a linear objective function subject to a set of non-linear constraints resulting from the measurement errors. Primal-dual logarithmic barrier path following method is applied in solving the constrained non-quadratic state estimation problem. This approach is computationally extremely useful because the principal computational step in solving the symmetric positive semi-definite system is identical to that of solving unconstrained weighted least squares (WLS) problem. Consequently, this method is implemented using modified WLS MATLAB software.

The paper is organized as follows. Section 2 presents Tanzanian power system network generation and high voltage transmission system status. The system is used as a case study in testing the non-quadratic state estimation algorithm. Section 3 gives material and method followed by problem formulation in which measurement model, least absolute value (LAV) state estimation formulation, nonquadratic constrained state estimation problem and algorithm are presented.

In section 4 a method of solving the non-quadratic estimation problem using primal-dual logarithmic barrier path following method is presented. Section 5 presents input data, simulation procedures and results. Section 6 discusses the obtained results and section 7 concludes the paper.

\section{Tanzanian System Network}

\subsection{Generation}

The Tanzanian power system comprises of hydro, thermal and isolated thermal generation plants [16]. The hydro system is comprised of 6 plants with a total nameplate of $561 \mathrm{MW}$ (See Table 1). The installed capacity of thermal generating plants totals $453.6 \mathrm{MW}$. The installed capacity of isolated thermal generating plants totals $38.45 \mathrm{MW}$. Currently, the total nameplate capacity is 1,053.05 MW [16]. The demand for electricity in Tanzania is growing at a relatively fast rate (See Table 4). The annual average load growth rate between 1990 and 1998 was 5 percent; the average load growth rate between 2003 and 2006 has been above 11 percent [17]. This load growth rate has been achieved despite long period of load shedding due to drought and inadequate water and rainfall in the main hydropower reservoirs and their catchments areas.

Tanzania Electric Supply Company Limited (TANESCO) owns all of the hydro generating plants in the country and some of the thermal generating plants, although there are some independent power producers (IPPs) owned by private operators. Tables 1 and 2 show the installed grid connected generation capacities for the country. The system presently consists of an interconnected grid and several isolated systems. The model of the interconnected grid is shown in Figure 1. The interconnected system consists of hydro and thermal generating plants providing power to Cities, Municipals and Townships.

Table 1. Installed hydro grid generation capacity

\begin{tabular}{llll}
\hline Plant Name & Fuel Type & Installed Capacity [MW] & Ownership \\
\hline Kihansi & Hydro & 180.00 & TANESCO \\
Kidatu & Hydro & 204.00 & TANESCO \\
Mtera & Hydro & 80.00 & TANESCO \\
NPF & Hydro & 68.00 & TANESCO \\
Hale & Hydro & 21.00 & TANESCO \\
NYM & Hydro & 08.00 & TANESCO \\
TOTAL & & 561.00 & \\
\hline
\end{tabular}

Table 2. Installed thermal grid generation capacity

\begin{tabular}{llll}
\hline Plant Name & Fuel Type & Installed Capacity [MW] & Ownership \\
\hline Songas & Natural gas & 202.00 & Private \\
Ubungo & Natural gas & 102.00 & TANESCO \\
IPTL & HFO & 103.00 & Private \\
Dodoma & IDO & 07.44 & TANESCO \\
Mbeya & IDO & 13.90 & TANESCO \\
Mwanza & IDO & 12.50 & TANESCO \\
Musoma & IDO & 02.56 & TANESCO \\
Tabora & IDO & 10.20 & TANESCO \\
TOTAL & & 453.60 & \\
\hline
\end{tabular}

Source: Economic Survey Report: 2007 and 2009 IDO - Industrial Diesel Oil, HFO- Heavy Fuel Oil 


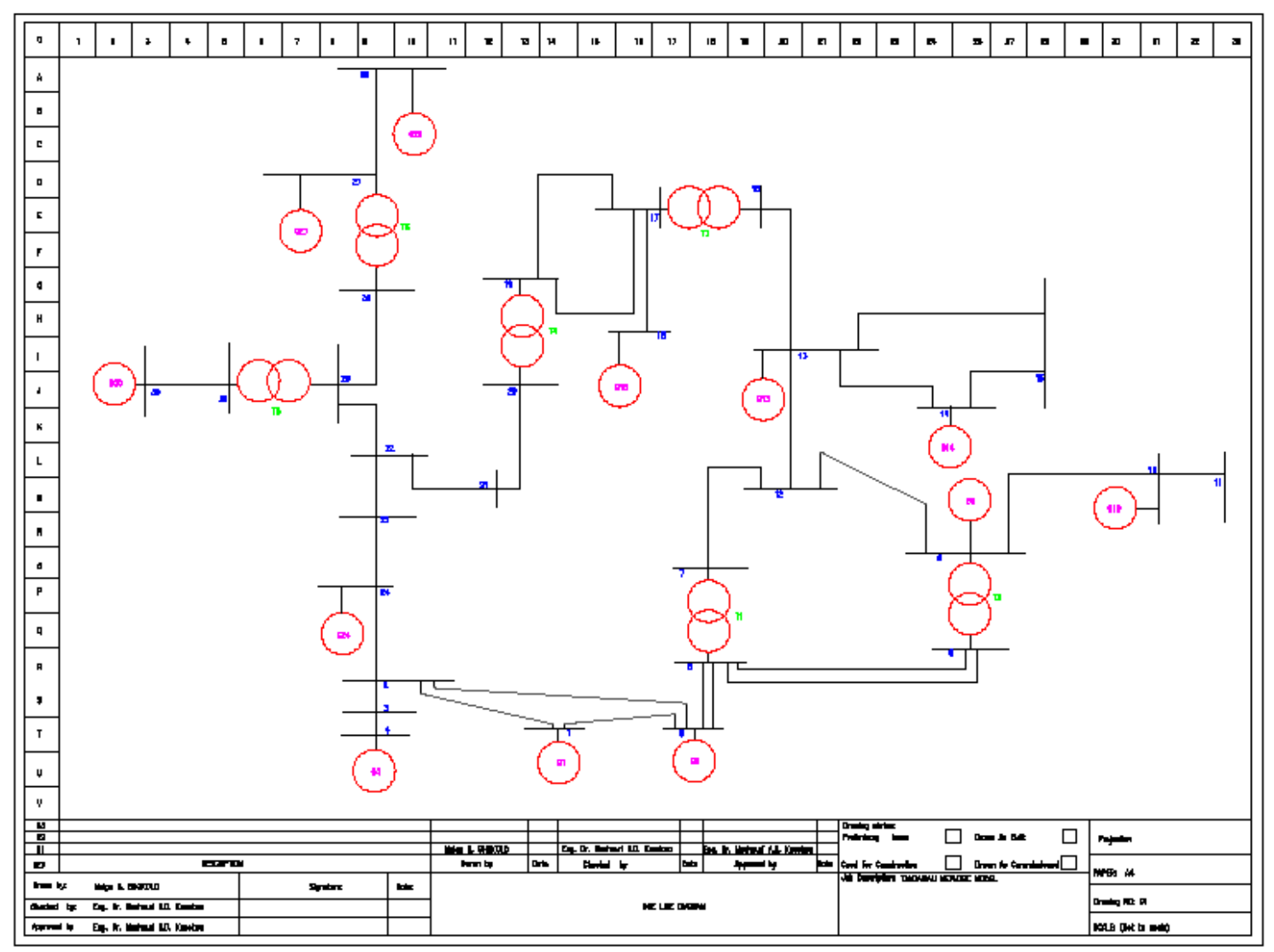

Figure 1. Tanzanian network model

\subsection{Transmission Network}

TANESCO owns high voltage and low voltage transmission and distribution lines of different voltage levels scattered all over the country. The high voltage transmission lines (See Table 3) are estimated to comprise of 2,624.36 km of system voltage $220 \mathrm{kV} ; 1,441.50 \mathrm{~km}$ of $132 \mathrm{kV}$ and $486.00 \mathrm{~km}$ of $66 \mathrm{kV}$, totalling to $4,551.86 \mathrm{~km}$ by the end of December 2006 [16]. High voltage transmission lines use pylons made of steel. Almost all HV transmission lines are radial single circuit lines. The country power system is alternating current (AC) and the system frequency is $50 \mathrm{~Hz}$. The TANESCO grid comprises of: South-West grid, NorthWest grid and North-East grid. South-East grid is still under planning stage.

South-West grid mostly of $220 \mathrm{kV}$ connects: UbungoMorogoro-Kidatu-Kihansi-Iringa-Mufindi-Mbeya. NorthWest grid connects: Ubungo-Morogoro-Kidatu-KihansiIringa-Mtera-Dodoma-Singida-Shinyanga-Mwanza $(220 \mathrm{kV})$; Mwanza- Musoma (132 kV)-Shinyanga- Tabora $(132 \mathrm{kV})$ North-East grid connects: Ubungo-Tegeta-Zanzibar $(132 \mathrm{kV})$; Ubungo-Chalinze- Hale-NPF-Tanga (132 kV); Chalinze Moshi - Arusha (132 kV); NYM - Moshi (66 kV); ArushaBabati-Singida $(220 \mathrm{kV})$.

\section{Material and Method}

Electrical data used in this study were obtained from Tanzania Electric Supply Company Limited (TANESCO). A computer software programme, MATLAB was used to determine the state vector of the Tanzania Power Electrical Network.

\subsection{Problem Formulation}

\subsubsection{Measurement Model}

The mathematical measurement model of state estimation is based on the mathematical relations between the measurement and the state vector given by:

$$
z=h(x)+r
$$

Where

$z \in \mathfrak{R}^{m x 1}$ Is the vector of measurements i.e. the voltages, injection powers and flow powers

$x \in \mathfrak{R}^{2 N-1}$ is the vector of state variables

$h(.) \in \mathfrak{R}^{m \times 1}$ is the non-linear function relating the measurement to state vector

$r \in \mathfrak{R}^{m \times 1}$ is the measurement residual vector

$N$ is the number of buses

$m$ is the number of measurements

$n=2 N-1$ is the number of state vector components 
The measurement system consists of real and reactive power injections, real and reactive line power flows and bus voltage magnitude.

\subsection{Least Absolute Value (LAV) State Estimation Criterion}

The least absolute value (LAV) state estimation problem criterion is formulated as:

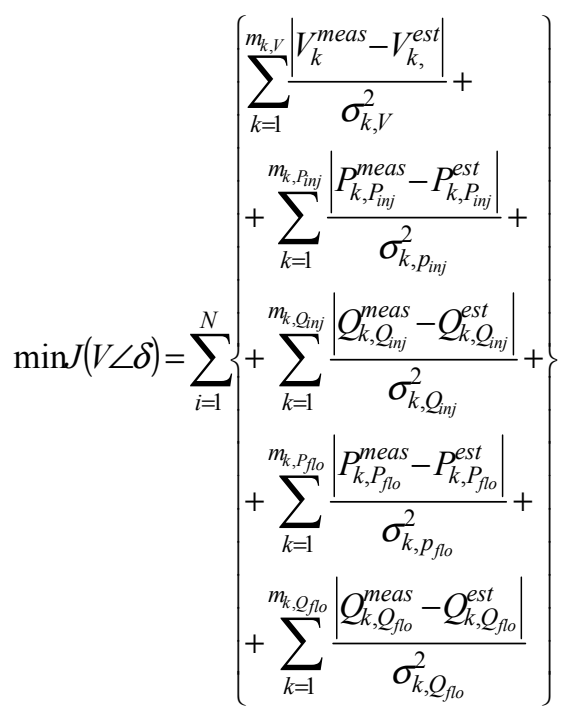

The objective of the estimator is to minimize the errors in order to get a best estimate of the system. In this way equation (2) is transformed into:

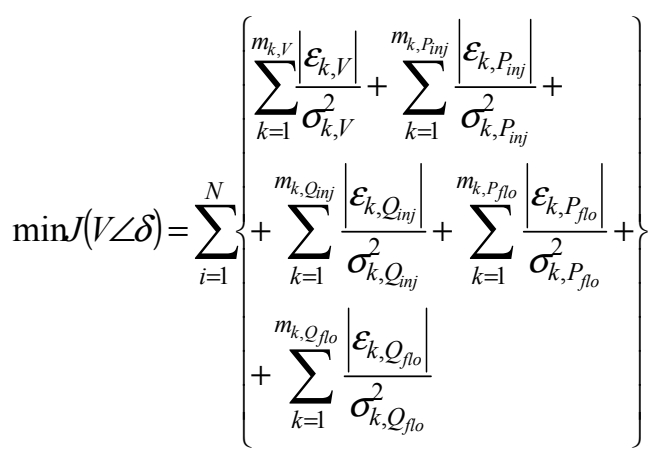

Where

$m_{k, V} \in \mathfrak{R}^{N}$ is the number of voltage magnitude measurement

$m_{k, P_{i n j}} \in \mathfrak{R}^{N}$ is the number of real power injection measurement?

$m_{k, Q_{i n j}} \in \mathfrak{R}^{N}$ is the number of reactive power injection measurement

$m_{k, P_{f l o}} \in \mathfrak{R}^{2 N}$ is the number of real power flows measurements

$m_{k, Q_{f l o}} \in \mathfrak{R}^{2 N}$ is the number of reactive power flows measurements

$\varepsilon_{k, V}:$ is the error corresponding to voltage magnitude $\varepsilon_{k, P_{i n j}}$ is the error corresponding to real power injection

$\mathcal{E}_{k, Q_{i n j}}$ is the error corresponding to reactive power injection

$\mathcal{E}_{k, P_{f l o}}$ is the error corresponding to real power flows

$\varepsilon_{k, Q_{f l o}}$ is the error corresponding to reactive power flows

$\delta$ : Voltage angle (unknown variable)

$\sigma:$ Standard deviation

$J$ : Objective function

The state estimator needs a set of analogue measurements and system topology to estimate the system state. The minimal measurement number required is equal to $(2 \mathrm{~N}-1)$ the dimension of the state vectors. Hence, the critical number of real and reactive measurement pair is $(\mathrm{N}-1)$ with addition of voltage magnitude measurement.

\subsection{Constrained State Estimation Problem}

The non-quadratic constrained state estimation problem is formulated by including equality and inequality constrains existing in the system. Equality constraints are target values used in load flow analysis and are included in power system state estimation in order to restore observability to those parts of the power system network which are permanently or temporarily unobservable. Equality constraints can be treated as pseudo-measurements with relative high weights [18].The equality constraints are the power balance equations which are described in Momoh [19] as:

$$
\begin{gathered}
P_{p}-V_{p} \sum_{q=1}^{N} V_{q}\left(G_{p q} \cos \delta_{p q}+B_{p q} \sin \delta_{p q}\right)=0 \\
p=\overline{1, N} \\
Q_{p}-V_{p} \sum_{q=1}^{N} V_{q}\left(G_{p q} \sin \delta_{p q}-B_{p q} \cos \delta_{p q}\right)=0 \\
p=\overline{1, N}
\end{gathered}
$$

Where

$p$ is not a slack bus, and $\delta_{\text {slack }}=0$

$P_{p}, Q_{p}$ is the real and reactive power injection at bus $\mathrm{p}$

$V_{p} \angle \delta_{p}$ is the voltage at bus $\mathrm{p}$

$\delta_{p q}=\delta_{p}-\delta_{q}$

$G_{p q}+j B_{p q}$ Are the corresponding elements in system bus admittance matrix

The power injection at bus $\mathrm{p}$ is defined as

$$
\begin{gathered}
P_{p}=P_{G p}-P_{L p} \\
Q_{p}=Q_{G p}-Q_{L p}
\end{gathered}
$$


Where

$P_{G p}, Q_{G p}$ Are the real and reactive power generation at bus $p$, while $P_{L p}, Q_{L p}$ are the real and reactive load power at bus $p$. Bus voltage magnitude including the slack bus, and bus voltage angles except the slack bus where $\delta_{\text {slack }}=0$ are taken as state vector $x$.

Inequality constraints are limits such as minimum and maximum reactive power generation, transformer tap and phase-shift limit

The constraints are initially relaxed as one approach to the solution and those constraints that are violated are enforced on the corresponding limits either as equality constraints with relative high weights. Interior point methods have been suggested in relaxing the constraints as proposed in [20]

Therefore, the aim of this paper is to minimize the errors given in (3) by considering all constraints existing in the system network. Normally, in the weighted least squares (WLS) estimators, the influence of a measurement on the state estimate increases with the size of its residual while non-quadratic estimators (LAV/WLAV) are designed to bound the influence of large residuals on state estimation with prediction that these residuals correspond to gross error measurements. With this idea in mind, in this paper a nonquadratic criterion is used in developing the method and algorithm for solution of estimation problem.

In this way the non-quadratic constraint weighted least absolute value (WLAV) state estimation is formulated as follows:

$$
\begin{aligned}
& \min \left[\operatorname{diag}\left(R^{-1}\right)\right]^{T}|z-h(x)| \\
& \text { Subject to }\left\{\begin{array}{l}
\varepsilon=z-h(x) \\
g(x)=0 \\
\varepsilon \geq 0
\end{array}\right.
\end{aligned}
$$

Where

$\operatorname{diag}\left(R^{-1}\right)=W$ is the weighting factor

$|z-h(x)|=\varepsilon \in \mathfrak{R}^{m x 1}$ is the measurement error vector

The measurement error vector is of the whole system as given by equation (3).

$g:$ is the non-linear vector function of the equality constraints.

Since it is difficult to solve Eqns (8) and (9) directly, the problem is transformed to the following equivalent problem as:

$$
\begin{aligned}
& \min W^{T} \varepsilon \\
& \text { s.t. }\left\{\begin{array}{l}
r-\varepsilon \leq 0 \\
-r-\varepsilon \leq 0 \\
g(x)=0 \\
r-z+h(x)=0 \\
\varepsilon \geq 0
\end{array}\right.
\end{aligned}
$$

The solution technique employed is solving the nonquadratic constrained estimation problem is a primal-dual logarithmic barrier path following interior point method.

\section{Solution Method}

\subsection{Primal-Dual Interior Point Methods}

Interior point methods (IPMs) for non-linear programming problems have been studied since the early 1950s by Fiacco and McCormick [21]. Interest in interior point method was rekindled by introduction of projective method by Karmarkar in 1984 [22]. Karmarkar's method was equivalent to an interior point method known as the logarithmic barrier function method. In this method, the inequality non-linear constraints in (10) can be converted to equality non-linear constraints by adding non-negative slack variable vectors $(u$, $l \geq 0)$.

To ensure that these slack variable vectors will remain positive, the logarithmic barrier function is appended to the objective function of eqn (10). Then the state estimation problem (10) is transformed to a problem with equality constraints (11), which can be written as:

$$
\begin{gathered}
\min W^{T} \varepsilon-\mu \sum_{k=1}^{m}\left(\ln u_{k}+\ln l_{k}\right) \\
\text { s.t. }\left\{\begin{array}{l}
-\varepsilon-r+u=0 \\
-\varepsilon+r+l=0 \\
g(x)=0 \\
r-z+h(x)=0 \\
(\varepsilon, u, l) \geq 0
\end{array}\right.
\end{gathered}
$$

Where

$\mu>0$ is the barrier parameter. It value is forced to decrease towards zero as the iterations progress.

$m$ is the number of rows of measurement vector $z$

$u^{k}, l^{k}$ : are the $k$ th elements of the slack variable vectors $u$ and $l$

The Lagrangian function of the problem (11) is defined as:

$$
\begin{array}{rl}
L=W^{T} & \mathcal{E}-\sum_{k=1}^{m}\left(\ln u_{k}+\ln l_{k}\right)-\lambda^{T}[-\mathcal{E}-r+u]- \\
& -\beta^{T}[-\mathcal{E}+r+l]-\eta^{T}[r-z+h(x)]-\gamma^{T} g(x)
\end{array}
$$

Where

$\lambda, \beta, \eta$, and $\gamma$ are vectors of Lagrange's multipliers

The Karush-Kuhn-Tucker (KKT) first order necessary conditions for an optimal solution of the sub-problems of (12) can be expressed in terms of a stationary point of the Lagrangian function are given by

$$
\begin{gathered}
\nabla_{u} L=-\mu U^{-1} e-\lambda=0 \\
\nabla_{l} L=-\mu L^{-1} e-\beta=0
\end{gathered}
$$




$$
\begin{gathered}
\nabla_{\varepsilon} L=W+\lambda+\beta=0 \\
\nabla_{\lambda} L=\varepsilon+r-u=0 \\
\nabla_{\beta} L=\varepsilon-r-l=0 \\
\nabla_{r} L=\lambda-\beta-\eta=0 \\
\nabla_{\gamma} L=-g(x)=0 \\
\nabla_{\eta} L=-r+z-h(x)=0 \\
\nabla_{x} L=-H^{T} \eta-G^{T} \gamma=0 \\
(\lambda, \beta) \geq 0
\end{gathered}
$$

Where

$\mathrm{H}$ and $\mathrm{G}$ are Jacobian matrices of $h(x), g(x)$.

$\mathrm{U}$ and $\mathrm{L}$ are diagonal matrices defined by slack variables $u$ and $l$, respectively given by equations (22) and (23) as:

$$
\begin{aligned}
U & =\left[\begin{array}{cccc}
u_{1} & 0 & \cdots & 0 \\
0 & u_{2} & \cdots & 0 \\
0 & 0 & \ddots & 0 \\
0 & 0 & 0 & u_{m}
\end{array}\right] \\
L & =\left[\begin{array}{llll}
l_{1} & 0 & \cdots & 0 \\
0 & l_{2} & \cdots & 0 \\
0 & 0 & \ddots & 0 \\
0 & 0 & 0 & l_{m}
\end{array}\right]
\end{aligned}
$$

Let $e=[1,1,1]^{T}$ : a vector with all its elements equals to one.

The KKT non-linear equations can be solved using different methods. They can be solved either as all equations together or by reducing them by elimination of variables. In this paper, the equations are solved iteratively using the Newton-Raphson method. In this method, the following linearizing approximations are made at each iteration.

$$
\begin{aligned}
& h(x) \approx h\left(x^{k}\right)+H \Delta x \\
& g(x) \approx g\left(x^{k}\right)+G \Delta x \\
& U^{-1} e \approx\left(U^{k}\right)^{-1} e-\left(U^{k}\right)^{-2} d u \\
& L^{-1} e \approx\left(L^{k}\right)^{-1} e-\left(L^{k}\right)^{-2} d l \\
& H \approx H\left(x^{k}\right) \\
& G \approx G\left(x^{k}\right)
\end{aligned}
$$

After these approximations are made and $d u$ and $d l$ are eliminated, the following system of equations results:

$$
\left.\left[\begin{array}{ccc}
D & 0 & H \\
0 & 0 & G \\
2 H^{T} & -G^{T} & 0
\end{array}\right] \begin{array}{c}
\beta \\
\gamma
\end{array}\right]=\left[\begin{array}{c}
-T R^{-1} \\
-g\left(x^{k}\right) \\
-H^{T} R^{-1}
\end{array}\right]
$$

Where

$\mathrm{D}$ is a diagonal matrix given by

$$
\begin{gathered}
D=\operatorname{diag} \frac{0.5}{\mu}\left\{\left(U^{k}\right)^{2}+\left(L^{k}\right)^{2}\right\} \\
T=\frac{0.5}{\mu}\left(U^{k}\right)^{2}
\end{gathered}
$$

The solution of Eqn (25) is used to calculate the direction of changes in $\beta$, fand $x$. It may not be possible to take a full Newton-Raphson step without violating the inequality constraints [23-24]. Hence, the new values of $\beta$, $\tau$, and $x$ are computed from:

$$
\begin{aligned}
& x^{k+1}=x^{k}+\alpha_{P} \Delta x \\
& \beta^{k+1}=\beta^{k}+\alpha_{D}\left(\beta-\beta^{k}\right) \\
& \gamma^{k+1}=\gamma^{k}+\alpha_{D}\left(\gamma-\gamma^{k}\right)
\end{aligned}
$$

$\alpha_{P}$ and $\alpha_{D}$ are scalars known as primal and dual step length. An advantage of using primal-dual method is that of using two step lengths, one for primal variables and the other for dual variables

In practice equality constraint measurement are error free, in this way the equality constraints component in equation (25) is neglected; this transforms the equation into

$$
\left[\begin{array}{cc}
D & H \\
2 H^{T} & 0
\end{array}\right]\left[\begin{array}{c}
\beta \\
\Delta x
\end{array}\right]=\left[\begin{array}{c}
-T W \\
-H^{T} W
\end{array}\right]
$$

$\Delta x$ can be computed from the following equation

$$
\begin{gathered}
2 H^{T} D^{-1} H \Delta x=2 H^{T} D^{-1} T W-H^{T} W \\
\Delta x=\left[2 H^{T} D^{-1} H\right]^{-1}\left(2 H^{T} D^{-1} T W-H^{T} W\right)
\end{gathered}
$$

\subsection{Updating and Adjusting the Variables}

Assuming that an initial starting point in the interior of the feasible region is known, and then the solution is updated in this way:

$$
\begin{aligned}
& x^{k+1}=x^{k}+\alpha_{P} d x \\
& u^{k+1}=u^{k}+\alpha_{P} d u \\
& l^{k+1}=l^{k}+\alpha_{P} d l \\
& \lambda^{k+1}=\lambda^{k}+\alpha_{D} d \lambda \\
& \beta^{k+1}=\beta^{k}+\alpha_{D} d \beta
\end{aligned}
$$

The step length $\alpha$ is choosen such that the solution remains within the feasible region i.e. $u>0$ and $l>0$. In order to 
keep

$$
\begin{aligned}
& u^{k+1} \geq 0: \\
& u^{k+1}=u^{k}+\alpha_{u} d u>0
\end{aligned}
$$

Then

$$
\alpha_{u}=\min \left\{-\frac{u^{k}}{|d u|}, d u<0\right\}
$$

Similarly

To keep

$$
\begin{aligned}
& l^{k+1} \geq 0: \\
& l^{k+1}=l^{k}+\alpha_{l} d l>0
\end{aligned}
$$

Then

$$
\alpha_{l}=\min \left\{-\frac{l^{k}}{|d l|}, d l<0\right\}
$$

In this way the step length is choosen using the following condition

$$
\alpha=\tau\left(1, \alpha_{u}, \alpha_{l}\right)
$$

$\tau$ is a scalar constant. The scalar constant value is set at 0.9995 in order to prevent the state estimation to be close to the feasible boundary. In the same way step length $\alpha$ is appropriately choosen in order to make $x^{k+1}$ remains interior to the feasible region. The iteration is performed until the norm [25] of $\Delta x$ becomes less than the pre-defined tolerance i.e.

$$
\|\Delta x\| \leq \varepsilon_{t o l}
$$

However, care should be taken when adjusting the barrier parameter because the parameter is linked to diagonal matrix $D$. if barrier parameter becomes zero, the diagonal matrix $D$ becomes singular and the whole matrix of equation (30) becomes singular and the solution cannot be found. Therefore, the barrier parameter should be appropriately adjusted and it should be close to zero but not equal to zero when $x$ approaches the optimal solution. At each iteration the complementary gap is used to adjust the barrier parameter and is choosen as proposed in [15] i.e.

$$
\mu=\frac{\lambda^{T} u+\beta^{T} l}{2 m}
$$

\subsection{The Algorithm}

The complete non-quadratic state estimation algorithm which uses primal-dual logarithmic barrier method in solving the state estimation problem is presented as follows. The algorithm has two parts. The first part is concerned with initialization procedure. Initial values are obtained from load flow results. The second part deals with computation of variables and implementation of the algorithm. The full algorithm is given as:

PART I: Initialize:

Initialize $k=0$.

$x^{0}=$ flat start using values obtained from the load flow program

$$
\begin{aligned}
& r^{0}=z-h\left(x^{0}\right) \\
& \varepsilon^{0}=\mid z-h\left(x^{0}\right) / \\
& u^{0}=\varepsilon^{0}+r^{0} \\
& l^{0}=\varepsilon^{0}-r^{0} \\
& \gamma=0 ; \tau=0, \lambda=-0.5 \varepsilon, \beta=-0.5 \varepsilon
\end{aligned}
$$

PART II: Calculation and implementation

i Calculate the Jacobian matrix $\mathrm{H}$

ii Calculate the transpose matrix $\mathrm{H}^{\mathrm{T}}$

iii Calculate the complementary gap

iv Calculate the barrier parameter $(\mu)$

$\mathrm{v}$ Calculate D

vi Calculate $d u=\varepsilon+r-u^{k}$

vii Calculate $d l=\varepsilon-r+l^{k}$

viiiCalculate $l^{k}$ and $u^{k}$

ix Calculate $r^{k}=z-h\left(x^{k}\right)$

$\mathrm{x}$ Solve $\Delta x$

xi Calculate step length

xii Update $x^{k+1}=x^{k}+\alpha \Delta x$

xiiiCheck if $/ z-h\left(x^{k+1}\right) /<0.0001$ if yes STOP. Otherwise go to III

xiv Update $\lambda$ and $\beta$

$\mathrm{xv}$ Update $u$ and $l$

xvi Check convergence criteria

If optimum TERMINATE the procedure. Otherwise update $k=k+1$ and go to III

\section{Simulation and Results}

\subsection{Input Data}

Voltage magnitude, real and reactive power injections, real and reactive power flows models are used for solving the state estimation problem. These models are written as:

$$
\begin{array}{r}
z_{i}=V_{i}+\varepsilon_{i, V} \quad z_{i} \in \mathfrak{R}^{N} i=\overline{1, N} \\
z_{i, \text { inj }}=h_{i, \text { inj }}(V \angle \delta)+\varepsilon_{i, i n j} \\
z_{i} \in \mathfrak{R}^{2 N} \quad i=\overline{1, N} \\
z_{i, \text { flow }}=h_{i, \text { flow }}(V \angle \delta)+\varepsilon_{i, \text { flow }} \\
z_{i} \in \mathfrak{R}^{m_{\text {flow }}} \quad i=\overline{1, m_{\text {flow }}}
\end{array}
$$

Voltage magnitude, real and reactive power injections, real and reactive power flows measurements are obtained from the load flow program and are accepted as true measurement values of the system.

Input data for simulation of the developed algorithm is given in Tables 3 and 4 . Table 3 gives transmission line data of the Tanzanian Power System Network. Table 4 gives load 
demand and generation of each bus. Input data for IEEE 14, IEEE30 bus test systems are obtained from [26].

Table 3. Line data 30-Bus Tanzania System Network

\begin{tabular}{|c|c|c|c|c|}
\hline From & To & Impedance & $\begin{array}{l}\text { Half of line } \\
\text { charging }\end{array}$ & $\begin{array}{l}\text { Tap ratio } \\
\text { setting }\end{array}$ \\
\hline 1 & 2 & $0.012+\mathrm{j} 0.081$ & $0.00+\mathrm{j} 0.065$ & 1.00 \\
\hline 2 & 3 & $0.020+\mathrm{j} 0.111$ & $0.00+\mathrm{j} 0.085$ & 1.00 \\
\hline 1 & 5 & $0.039+\mathrm{j} 0.154$ & $0.00+\mathrm{j} 0.122$ & 1.00 \\
\hline 2 & 5 & $0.025+\mathrm{j} 0.136$ & $0.00+\mathrm{j} 0.010$ & 1.00 \\
\hline 2 & 24 & $0.016+\mathrm{j} 0.090$ & $0.00+\mathrm{j} 0.068$ & 1.00 \\
\hline 3 & 4 & $0.034+\mathrm{j} 0.019$ & $0.00+\mathrm{j} 0.143$ & 1.00 \\
\hline 5 & 6 & $0.014+\mathrm{j} 0.011$ & $0.00+\mathrm{j} 0.087$ & 1.00 \\
\hline 6 & 7 & $0.00+\mathrm{j} 0.274$ & $0.00+\mathrm{j} 0.00$ & 1.00 \\
\hline 6 & 8 & $0.018+\mathrm{j} 0.015$ & $0.00+\mathrm{j} 0.117$ & 1.00 \\
\hline 7 & 12 & $0.086+\mathrm{j} 0.196$ & $0.00+\mathrm{j} 0.020$ & 1.00 \\
\hline 8 & 9 & $0.00+\mathrm{j} 0.062$ & $0.00+\mathrm{j} 0.00$ & 1.00 \\
\hline 9 & 10 & $0.043+\mathrm{j} 0.098$ & $0.00+\mathrm{j} 0.00$ & 1.00 \\
\hline 9 & 12 & $0.010+\mathrm{j} 0.232$ & $0.00+\mathrm{j} 0.024$ & 1.00 \\
\hline 10 & 11 & $0.052+\mathrm{j} 0.030$ & $0.00+\mathrm{j} 0.00$ & 1.00 \\
\hline 12 & 13 & $0.018+\mathrm{j} 0.418$ & $0.00+\mathrm{j} 0.043$ & 1.00 \\
\hline 13 & 14 & $0.009+\mathrm{j} 0.027$ & $0.00+\mathrm{j} 0.00$ & 1.00 \\
\hline 13 & 15 & $0.063+\mathrm{j} 0.014$ & $0.00+\mathrm{j} 0.00$ & 1.00 \\
\hline 14 & 15 & $0.049+\mathrm{j} 0.014$ & $0.00+\mathrm{j} 0.00$ & 1.00 \\
\hline 13 & 16 & $0.026+\mathrm{j} 0.597$ & $0.00+\mathrm{j} 0.062$ & 1.00 \\
\hline 16 & 17 & $0.00+\mathrm{j} 0.7373$ & $0.00+\mathrm{j} 0.00$ & 1.00 \\
\hline 17 & 19 & $0.036+\mathrm{j} 0.716$ & $0.00+\mathrm{j} 0.00$ & 1.00 \\
\hline 18 & 17 & $0.018+\mathrm{j} 0.037$ & $0.00+\mathrm{j} 0.00$ & 1.00 \\
\hline 20 & 19 & $0.00+\mathrm{j} 0.1416$ & $0.00+\mathrm{j} 0.00$ & 1.00 \\
\hline 20 & 21 & $0.023+\mathrm{j} 0.014$ & $0.00+\mathrm{j} 0.111$ & 1.00 \\
\hline 21 & 22 & $0.021+\mathrm{j} 0.131$ & $0.00+\mathrm{j} 0.100$ & 1.00 \\
\hline 22 & 23 & $0.033+\mathrm{j} 0.017$ & $0.00+\mathrm{j} 0.137$ & 1.00 \\
\hline 23 & 24 & $0.021+\mathrm{j} 0.012$ & $0.00+0.081$ & 1.00 \\
\hline 22 & 25 & $0.034+\mathrm{j} 0.188$ & $0.00+\mathrm{j} 0.143$ & 1.00 \\
\hline 25 & 26 & $0.022+\mathrm{j} 0.118$ & $0.00+\mathrm{j} 0.095$ & 1.00 \\
\hline 25 & 29 & $0.00+\mathrm{j} 0.160$ & $0.00+\mathrm{j} 0.00$ & 1.00 \\
\hline 26 & 27 & $0.00+\mathrm{j} 0.160$ & $0.00+\mathrm{j} 0.00$ & 1.00 \\
\hline 27 & 28 & $0.263+\mathrm{j} 0.597$ & $0.00+\mathrm{j} 0.061$ & 1.00 \\
\hline 29 & 30 & $0.021+\mathrm{j} 0.485$ & $0.00+\mathrm{j} 041$ & 1.00 \\
\hline
\end{tabular}

Table 4. Busdata 30 -Bus Tanzania System Network

\begin{tabular}{|c|c|c|c|c|}
\hline \multirow{2}{*}{ Bus No. } & \multicolumn{2}{|c|}{ Load demand } & \multicolumn{2}{|c|}{ Generation } \\
\hline & MW & MVAr & MW & MVAr \\
\hline 1 & - & - & - & - \\
\hline 2 & 06.20 & 01.60 & - & - \\
\hline 3 & 20.00 & 07.00 & - & - \\
\hline 4 & 27.00 & 07.80 & 14 & - \\
\hline 5 & - & - & 142.00 & - \\
\hline 6 & 18.00 & 09.10 & - & - \\
\hline 7 & 00.00 & 00.00 & - & - \\
\hline 8 & 233.10 & 45.10 & - & - \\
\hline 9 & - & - & 259.00 & - \\
\hline 10 & - & - & 100.00 & - \\
\hline 11 & 17.60 & 09.00 & - & - \\
\hline 12 & 12.00 & 02.50 & - & - \\
\hline 13 & - & - & 10.50 & - \\
\hline 14 & - & - & 68.00 & - \\
\hline 15 & 21.00 & 08.30 & - & - \\
\hline 16 & 23.10 & 09.00 & - & - \\
\hline 17 & 00.00 & 00.00 & - & - \\
\hline 18 & - & - & 03.60 & - \\
\hline 19 & 22.00 & 05.00 & - & - \\
\hline 20 & 00.00 & 00.00 & - & - \\
\hline 21 & 06.50 & 01.20 & - & - \\
\hline 22 & 05.00 & 01.40 & - & - \\
\hline 23 & 06.20 & 01.60 & - & - \\
\hline
\end{tabular}

\begin{tabular}{lllll}
\hline \multirow{2}{*}{ Bus No. } & \multicolumn{2}{l}{ Load demand } & Generation & \\
\cline { 2 - 5 } & MW & MVAr & MW & MVAr \\
\hline 24 & - & - & 74.00 & - \\
25 & 21.70 & 09.00 & - & - \\
26 & 29.70 & 09.60 & - & - \\
27 & - & - & 13.00 & - \\
28 & 11.50 & 05.00 & - & - \\
29 & 00.00 & 00.00 & - & - \\
30 & 05.40 & 01.50 & - & - \\
\hline
\end{tabular}

Table 5. Test systems

\begin{tabular}{llll}
\hline No. of buses & Ward Hale 6 & IEEE 14 & 30-Bus \\
\hline No. of lines & 7 & 20 & 33 \\
No. of measurements & 25 & 41 & 97 \\
Redundancy & 227.27 & 151.85 & 164.41 \\
\hline
\end{tabular}

\subsection{Simulation}

The prototype code of the non-quadratic constrained state estimation algorithm was implemented in MATLAB 7.1 and run on a personal computer having a $3.33 \mathrm{GHz}$ Pentium IV processor and $0.99 \mathrm{~GB}$ of RAM. Simulations were carried on using the IEEE 14, IEEE30 test systems and the 30-bus Tanzanian power system network model. The problem statistics are summarized in Table 5, where the number of buses, lines, total number of measurements and redundancies are given. The measurements were simply chosen such that the systems become numerically observable. Numerical observability was checked using the rank (.) function in MATLAB.

The measurements are simulated adding normally distributed random error to the load flow results. The following standard deviations of the measurements are used: $0.004,0.008$, and 0.01 p.u. standard deviations for voltage magnitudes, line flows and bus injection, respectively. To verify the accuracy of the resulting estimates, the following error criteria are calculated according to [12]

$$
\begin{gathered}
\Delta V_{r m s}=\frac{1}{N} \sqrt{\sum_{p=1}^{N}\left(V_{p}-V_{p}^{n o n}\right)} \\
\Delta \delta_{r m s}=\frac{1}{N} \sqrt{\sum_{p=1}^{N}\left(\delta_{p}-\delta_{p}^{n o n}\right)}
\end{gathered}
$$

Where

$V_{p}, \delta_{p}$ : Are the true (load flow solution) voltage magnitude and phase angle at bus $p$

$V_{p,}^{n o n}, \delta_{p}^{n o n}:$ Are the estimates obtained from the nonquadratic state estimator representing the voltage magnitude and phase angle at bus $p$.

\subsection{Simulation Results}

Voltage magnitude and voltage angle profiles are presented in Table 6. Figures 2 and 3 present voltage magnitudes and voltage angle profiles in graphical form. The estimation errors for voltage magnitudes and voltage angles are 
presented in Figures 4 and 5, respectively. Figure 6 shows upper $(u)$ and lower $(l)$ slack variable distribution in measurements and comparison of measurement residual (r) and measurement error ( $\mathcal{E}$ ) distribution is presented in Figure 7.

VOLT AGE MAGNIT UDE PROFILE-T ANZANIA NET WORK

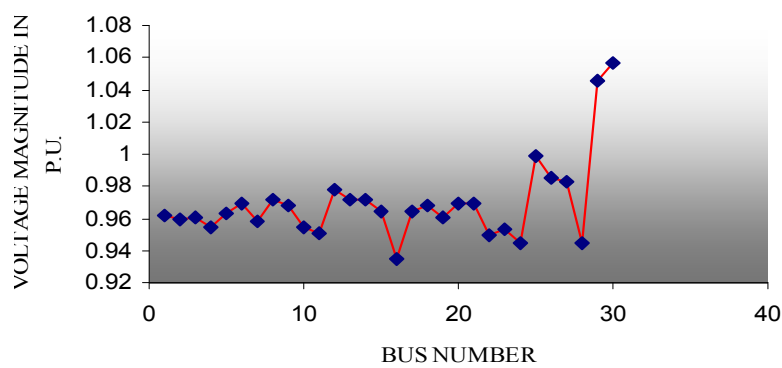

Figure 2. Voltage magnitude profile-Tanzania Network

VOLTAGE ANGLE PROFILE-TANZANIA NETWOK

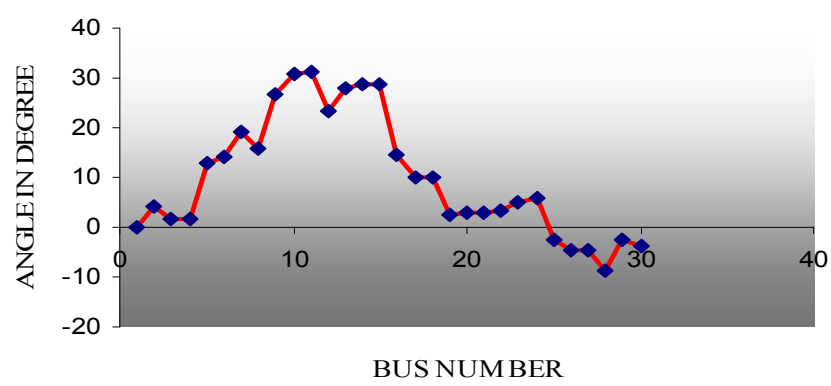

Figure 3. Voltage angle profile-Tanzania Network

VOLT AGE MAGNITUDE ERRORS-T ANZANIA NETWORK

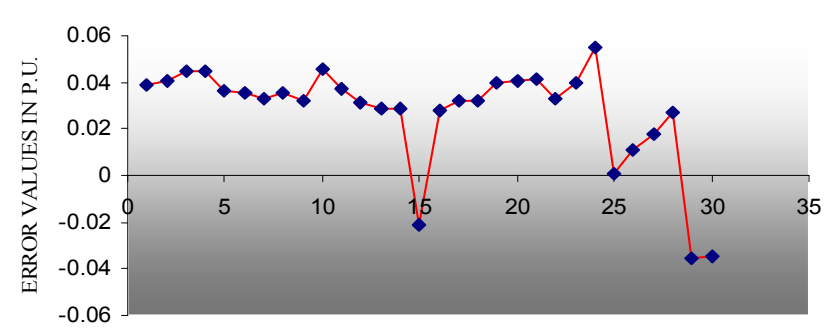

BUS NUMBER

Figure 4. Voltage magnitude errors-Tanzania Network

VOLTAGE ANGLE ERRORS-TANZANIA NETWORK

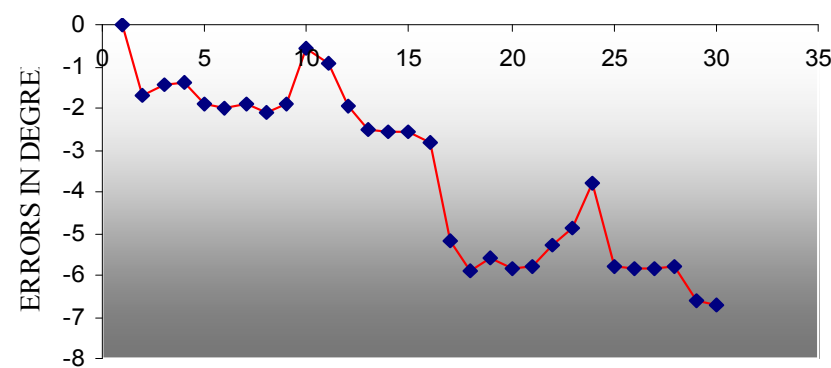

BUS NUMBER

Figure 5. Voltage angle errors-Tanzania Network
Table 6. Voltage mag. and Angle profiles of Tanzania System Network

\begin{tabular}{|c|c|c|c|c|c|}
\hline $\begin{array}{l}\text { Bu } \\
\text { s } \\
\text { No } \\
. \\
\end{array}$ & $\begin{array}{l}\text { Voltage } \\
\text { Magnitude[p } \\
\text {.u.] }\end{array}$ & $\begin{array}{l}\text { Voltage } \\
\text { Angle[Degr } \\
\text { ee] }\end{array}$ & $\begin{array}{l}\text { Bu } \\
\text { s } \\
\text { No } \\
\text {. } \\
\end{array}$ & $\begin{array}{l}\text { Voltage } \\
\text { Magnitude[p } \\
\text {.u.] }\end{array}$ & $\begin{array}{l}\text { Voltage } \\
\text { Angle[Degr } \\
\text { ee] }\end{array}$ \\
\hline 1 & 00.9614 & 00.0000 & 16 & 00.9350 & 14.4283 \\
\hline 2 & 00.9594 & 04.2278 & 17 & 00.9646 & 09.9308 \\
\hline 3 & 00.9608 & 01.7868 & 18 & 00.9675 & 09.9496 \\
\hline 4 & 00.9548 & 01.7692 & 19 & 00.9605 & 02.4502 \\
\hline 5 & 00.9636 & 13.1024 & 20 & 00.9696 & 02.9531 \\
\hline 6 & 00.9687 & 14.3279 & 21 & 00.9697 & 02.9651 \\
\hline 7 & 00.9579 & 19.2351 & 22 & 00.9494 & 03.4359 \\
\hline 8 & 00.9716 & 15.8562 & 23 & 00.9533 & 05.2061 \\
\hline 9 & 00.9675 & 26.6922 & 24 & 00.9450 & 05.8016 \\
\hline 10 & 00.9545 & 30.7587 & 25 & 00.9989 & -02.6612 \\
\hline 11 & 00.9511 & 31.0538 & 26 & 00.9854 & -04.5657 \\
\hline 12 & 00.9775 & 23.1554 & 27 & 00.9823 & -04.4707 \\
\hline 13 & 00.9715 & 27.9832 & 28 & 00.9443 & -8.6637 \\
\hline 14 & 00.9715 & 28.9300 & 29 & 01.0458 & -02.3242 \\
\hline 15 & 00.9649 & 28.5558 & 30 & 01.0568 & -03.7123 \\
\hline
\end{tabular}

The estimation errors for voltage magnitudes and voltage angles are presented in Figures 4 and 5, respectively. Figure 6 shows upper $(u)$ and lower $(l)$ slack variable distribution in measurements and comparison of measurement residual (r) and measurement error $(\mathcal{E})$ distribution is presented in Figure 7.
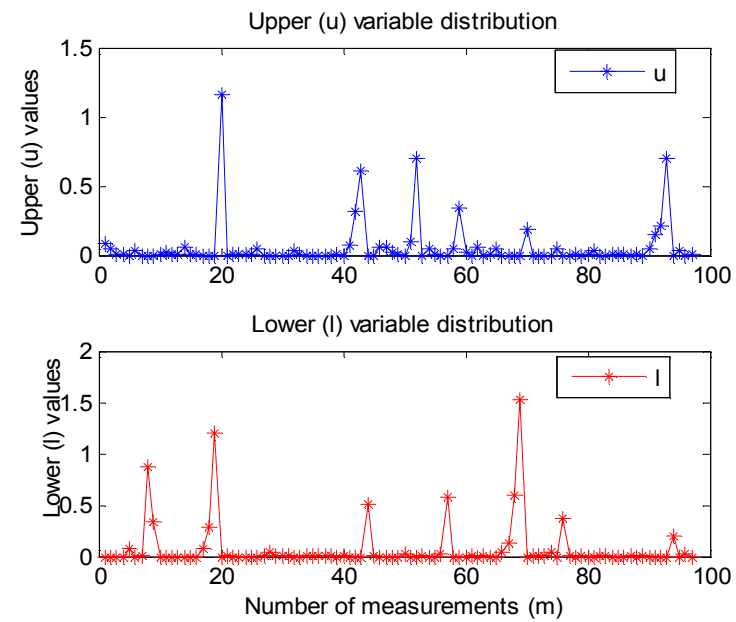

Figure 6. Upper (u) and lower (l) slack variable distributions
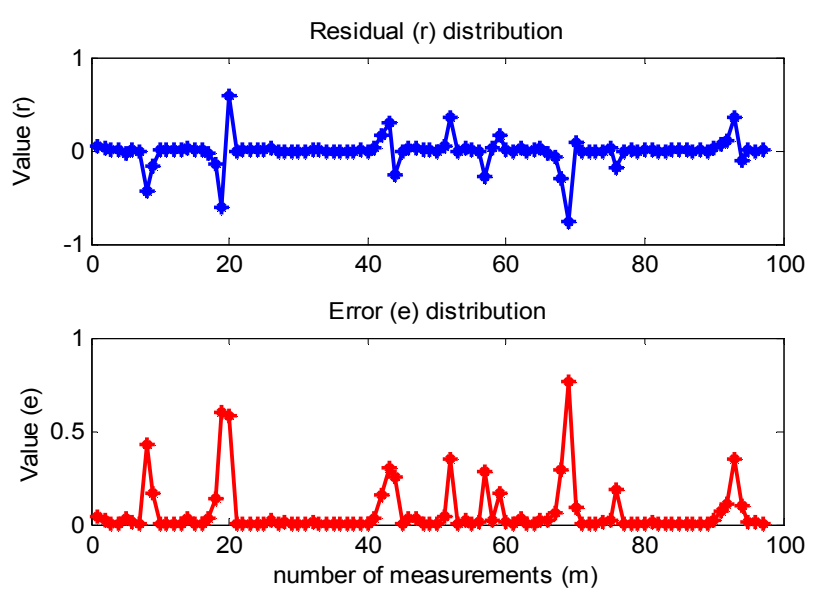

Figure 7. Comparison of residual (r) and error distribution 


\section{Discussion}

The following observations from simulation results can be made. The voltage magnitude and voltage angle profiles of the Tanzanian power system are within acceptable limits i.e. 0.95-1.10 per unit for voltage magnitude and $-35^{0}-+35^{0}$ degree for voltage angle. The power factor (pf) of the system is around $0.857\left(+31^{0}\right.$ degree $)$ which is the accepted operating value by TANESCO. Voltage magnitude estimation errors are within pre-defined range of $\pm 5 \%$.

The number of iterations is conditioned by the fact that not full Newton-Raphson step length $(\alpha)$ for primal variables is taken in order to remain in the feasible region. If in case measurements are acting in such way that some of constrains tend to be violated, the step length is only restricted to some iterations. The decrease of iterations number is achieved if more accurate initialization i.e. flats start initialization of state vector $x^{0}$ is applied.

\section{Conclusion}

Power system state estimation is a critical function in determining real-time model for interconnected system networks. In this environment, a real-time model is extracted at intervals from snapshots of real-time measurements. It is generally accepted that the ever expanding system networks demand network models that are more accurate and reliable than ever. This can only be achieved with robust state estimators that reliably deal with state and topology processing. With that in mind, this paper has presented development of a non-quadratic state estimation method and algorithm that incorporate equality and inequality constraints in its formulation. The simulation results demonstrate that the primal-dual logarithmic barrier interior point algorithm is a useful numerical tool to compute the state of an electrical power system network, when inequality constraints play the essential role in enhancing the reliability of the estimation results. Also, it is expected that the significant benefit could be gained from application of the constrained state estimation method and algorithm to the Tanzanian power system network.

\section{Acknowledgement}

I would like to thank the Tanzania Electric Supply Company Limited (TANESCO) for its cooperation and readiness to supply the most needed data to make this research work possible. Their support is gratefully acknowledged.

\section{References}

[1] F.F. Wu. Real-Time network security monitoring, assessment and optimization, $9^{\text {th }}$ Power Systems Computation Conference,Cascais, Portugal,31 August-4 September 1987: 83-100.

[2] A. Monticelli. State estimation in Electric Power Systems: A generalized approach. Kluwer Academic Publishers; Boston, 1999.

[3] N.H. Abbasy and S.M. Shahidehpour. Application of Nonlinear Programming in Power System State Estimation, Electric Power Systems Research, 12, 1987:41-50.

[4] R.E. Larson, W.F. Tinney, and J. Peschon, State Estimation in Power Systems, part I: Theory and feasibility. IEEE Transactions on Power Apparatus \& Systems; PAS-89(3), 1970: $345-352$

[5] F.C. Schweppe and J.Wildes. Power system Static State Estimation part I: exact model, IEEE Transactions on Power Apparatus \& Systems; Vol. PAS-89(1); 1970: 120-125

[6] F.C. Schweppe and D.B. Rom. Power System Static State Estimation, part II: approximate model, IEEE Transactions on Power Apparatus \& Systems; Vo. PAS-89, (1), 1970: 125-130

[7] F.C. Schweppe, Power System Static State Estimation part III: implementation, IEEE Transaction on Power Apparatus \& Systems; Vol. PAS-89(1), 1970: 130-135

[8] J.J. Allemong, L.Radu, and A.M. Sasson. A fast and reliable state estimation algorithm for AEP's new control centre, IEEE Transactions on Power Apparatus \& Systems; Vol. PAS101(3), 1982: 933-944

[9] L. Holten, A. Gjelsvick, S. Aam, F.F. Wu, and W.H.E. Liu, Comparison of different methods for state estimation, IEEE Transaction on Power Systems; 3, (4), 1988: 1798-1806

[10] A. Abur and A.G. Exposito, Power System State EstimationTheory and Implementations. New York: Marcel Dekker, 2004

[11] A. Abur and M. K. Celik, Least Absolute Value State Estimation with Equality and Inequality Constraints, IEEE Transactions on Power Systems; 8, (2), 1993: 680-686

[12] A. Abur and M.K. Celik, A fast algorithm for the Weighted Least Absolute Value State Estimation, IEEE Transactions on Power Systems; 6, (1), 1991: 1-8

[13] K.A. Clements, P.W. Davis, and K.D. Frey, An interior Point Algorithm for Weighted Least Absolute Value Power System State Estimation; IEEE/PES, 91-WM 235-2 PWRS, New York, 1991

[14] H. Singh and F.L. Alvarado, Weighted least Absolute Value State Estimation using Interior Point Methods. IEEE Transactions on Power Systems; 9(3), 1994: 1478-1484

[15] H. Wei, H. Sasaki, J. Kubokawa, and R. Yokoyama, An interior point method for power system weighted non-linear $\mathrm{L}_{1}$ norm static state estimation. IEEE Transaction on Power Systems; 13(2), 1998: 17-23

[16] http://www.tanesco.com (May 2012)

[17] http://www.nishati.go.tz (March 2012)

[18] F.F. Wu, and A. Monticelli, A critical review on external network modelling for on-line security analysis. International Journal of Electrical Power Engineering Systems, Vol.3, 1983: 222-235

[19] J.A. Momoh, Electric Power System Applications of Optimization, CRC Press, Taylor \& Francis Group, New York, 2009 
[20] K.A. Clements, P.W. Davis, and K.D. Frey, Treatment of inequality constraints in power system state estimation, IEEE Transactions on Power Systems, Vol. 10, 1995: 567-573

[21] A.V. Fiacco and G.P. McCormick, Non-linear Programming: Sequential Unconstrained Minimization Technique, John Wiley \& Sons, New York, 1968

[22] N.K. Karmarkar, A new polynomial-time algorithm for linear programming, Combinatorica 4, 1984: 183-295.

[23] K.A. Clements, P.W. Davis, and K.D. Frey, An efficient algorithm for computing the Weighted Least Absolute Value
Estimation in Power System State Estimation, "Proceeding of IFAC int. Symposium on Power System and Power Plant Control, Seoul Korea, August 22-25, 1989: 785-790

[24] K. Chiite and K.S. Swarup, Power System State Estimation using IP Barrier method, department of Electrical Engineering, Indian Institute of Technology Madras, 600036 INDIA, 2003: $1-6$

[25] Information on norms obtained from http://www.mathworld.wolfram.com/Norm.html

[26] http://www.ee.washington.edu/research/pstca 